\title{
Mímesis en el registro funerario orientalizante. Una propuesta de estudio
}

\author{
Imitation in protohistoric funerary context. \\ A proposal of analysis
}

\author{
Raquel Rodríguez MuÑOZ*
}

\begin{abstract}
RESUMEN
En este trabajo realizamos un análisis sobre el proceso de mímesis aplicado a un determinado objeto en el mundo orientalizante: el recipiente conocido como urna del tipo Cruz del Negro. Su adopción y asimilación, con carácter eminentemente funerario, por parte de las poblaciones locales asentadas en diversos puntos del sur peninsular, Extremadura y área de Levante, así como por parte de algunas comunidades fenicias en momentos posteriores, nos permite estudiar este complicado proceso de imitación desde varias perspectivas que resultan muy interesantes.
\end{abstract}

\section{PALABRAS CLAVE:}

Mímesis, urna Cruz del Negro, adopción, asimilación, poblaciones locales, comunidades fenicias, imitación.

\begin{abstract}
In this work we analyse imitation in the case of pottery containers of the "Cruz del Negro" type, commonly employed as burial jars. Its adoption and assimilation by local populations in the South, Extremadura and East of Iberian Peninsula, and its later use by Phoenicians, allow us to study this complex process of imitation from several points of view.
\end{abstract}

\section{KEYWORDS:}

Imitation, Cruz del Negro jar, adoption, assimilation, local populations, Phoenicians, imitation.

* Departamento de Prehistoria y Arqueología, Universidad de Valencia (Avda. Blasco lbáñez, 28. 46010 Valencia). E-mail: paureb@gmail.com.

Artículo basado en la comunicación leída el 19 de mayo de 2008, en la VII edición del Encuentro de Jóvenes Investigadores de Historia Antigua de la Universidad Complutense de Madrid. 


\section{INTRODUCCIÓN}

Hablar del registro funerario orientalizante es hablar de un tema extenso, complejo, que abarca muchos aspectos materiales como fiel reflejo de factores sociales, económicos y culturales; en definitiva, es tratar un conjunto de datos asociados directa o indirectamente con la muerte que pueden ser enfocados desde diversos puntos de vista. Por otro lado, hablar de registro funerario orientalizante es hablar de la mezcla de culturas que hacia comienzos del I milenio a.C. se produce en el Mediterráneo, así como de las nuevas realidades surgidas de la misma. Si a este tema tan amplio y complicado le añadimos el estudio del proceso cultural surgido en torno a la adopción de determinados aspectos, tanto materiales como relacionados con el comportamiento social, y la posterior imitación de modelos, así como la actualmente tan cuestionada aculturación que ello implica, necesitaríamos varios volúmenes para intentar acercarnos ligeramente a la génesis y desarrollo de este proceso tan interesante.

Por tanto, teniendo presentes estas cuestiones, resulta obvio que me centre principalmente en el sur de la Península lbérica, aunque haremos alusión a otras zonas, como el área extremeña y el Levante. Serán en estos contextos en los que se desarrolle una nueva realidad social, cuyo reflejo más palpable es el registro funerario, fruto de los contactos de los contingentes fenicios que se establecerán en el sur y este peninsular y de las diversas poblaciones locales que los habitaban.

\section{UN RECIPIENTE, DISTINTOS USOS}

Quiero hacer especial hincapié en los diversos enfoques de esta cuestión, que desde el punto de vista material puede ser entendida como el estudio de elementos tan significativos como los marfiles, la joyería, los ritos y pautas funerarias o la identificación de divinidades y cultos indígenas con foráneos, por ejemplo. En este sentido, planteo en mi comunicación una propuesta de estudio diferente, centrada en el recipiente conocido comúnmente como Urna del tipo de la Cruz del Negro, que debe ser entendida desde dos puntos de vista para tratar de comprender los procesos culturales asociados a la colonización y a la interacción entre sendas culturas: 1. La adopción y posterior asimilación de un objeto presumiblemente fenicio dentro del comportamiento funerario local. 2. La adopción de ese objeto, que se ha convertido en una seña de identidad de la nueva realidad funeraria indígena, por parte de algunos miembros foráneos ${ }^{1}$.

Estudios monográficos de este tipo de recipientes ya habían sido llevados a cabo por algunos autores (Aubet 1978: 267-87; Aranegui 1980: 99-118; García 1998: 115-19), en la mayoría de los casos desde la perspectiva indígena, como

1 En uso del término 'indígena' que haremos en estas páginas no responde a ninguna cuestión de tipo discriminatorio con respecto al término fenicio, foráneo o extranjero, sino como contraposición a los mismos, y como sinónimo de local. En este sentido, seguiremos la definición establecida por la RAE para el mismo: «Originario del país de que se trata». 
parte de ese nuevo espacio que surge tras la llegada de los fenicios a la Península Ibérica y ese objeto pasa a formar parte de esa nueva realidad socio-cultural, que tiene su mejor reflejo en el ámbito funerario, de donde proceden la mayoría de los hallazgos. En este sentido, lo que hemos echado en falta siempre es el análisis del uso de estos contenedores desde la óptica fenicia - una excepción sería el trabajo de García (1998)—, sobre todo si tenemos en cuenta que es escaso frente a su presencia en el ámbito indígena.

Cuando hace algún tiempo intentamos reabrir el debate en torno a esta cuestión (Rodríguez Muñoz 2006: 93-106), ya comentamos el posible origen oriental de tales objetos. Siguiendo a Belén y Pereira, apuntamos que los prototipos de las urnas Cruz del Negro podrían localizarse en Tell Qasile, Tell Abu Hawan y Beth Shemash, desde donde podrían haber cruzado a Chipre (Belén y Pereira 1985: 318). Ejemplos parecidos a los que luego se documentarán en Andalucía ${ }^{2}$ y Extremadura $^{3}$ los hallamos en Cartago (Cintas 1950: 153, 463; tabla III) así como en necrópolis centromediterráneas, como la de Mozia (Tusa 1978: 7-98). Observando los ejemplares hallados en estos cementerios pensamos que las producciones peninsulares pudieron haber sufrido una evolución propia, según se desprende de la arista en la parte central del cuello, mucho más marcada en los vasos hispanos, así como por llevar siempre dos asas, tener una mayor altura - los hallados en Mozia, sin duda los más parecidos, son, por norma general, ligeramente más pequeños - y presentar un galbo mucho más esférico y globular. Estas diferencias entre los ejemplares mediterráneos y los hispanos han permitido que algunos autores consideren a estos recipientes peninsulares como un «producto colonial» (Jiménez Ávila 2002: 147). El uso de este tipo de recipientes, urnas globulares con baquetón central y dos asas, estuvo dedicado fundamentalmente a la contención de las cenizas de los difuntos y en la Península lbérica su uso funerario se traspasó a las poblaciones locales.

Sin ánimo de entrar a valorar y debatir el origen fenicio o indígena de algunas necrópolis del valle del Guadalquivir, concretamente el cementerio de la Cruz del Negro, lo cierto es que, salvo algunas excepciones, el empleo de estos vasos se ha documentado en cementerios que están relacionados con las poblaciones locales asentadas en sus proximidades, por lo que podemos pensar que, tras su comercialización o intercambio, estos contenedores no perdieron su uso original.

El problema, desde nuestro punto de vista, surge cuando pretendemos acercarnos a la realidad funeraria fenicia, ya que nos encontramos con un panorama

2 Los ejemplares andaluces se han localizado en la necrópolis onubense de La Joya (Garrido 1970: 65-66; Garrido y Orta 1978: 24-39, 48-63; Orta y Garrido 1963: 10-36; figs. 4 y 5), en las necrópolis gaditanas de Mesas de Asta (González, Barrionuevo y Aguilar 1993: 215-37; Torres 1999: 65-66) y Las Cumbres (Ruiz Mata 1987: 158-60; Ruiz Mata y Pérez 1989: 287-95; 1995: 119-20), en los cementerios sevillanos de Campo de las Canteras (Belén, Gil, Lineros y Puya 1987: 543-44), Cruz del Negro (Gil y Puya 1995: 83-87), Setefilla (Aubet 1976: 16-17, 31), Angorrilla (Fernández y Rodríguez 2007: 83) y del Camino (Sánchez Andreu y Ladrón de Guevara 2000: 1895-1902), así como la necrópolis malagueña del Cortijo de las Sombras (Arribas y Wilkins 1969: 185-206). Para el caso de las necrópolis andaluzas estrictamente fenicias, véase una revisión en Rodríguez Muñoz (2006: 96-101).

3 En este sentido destaca la necrópolis de Medellín (Almagro 1991: 233-52; 2007; 2008: 631-54). 


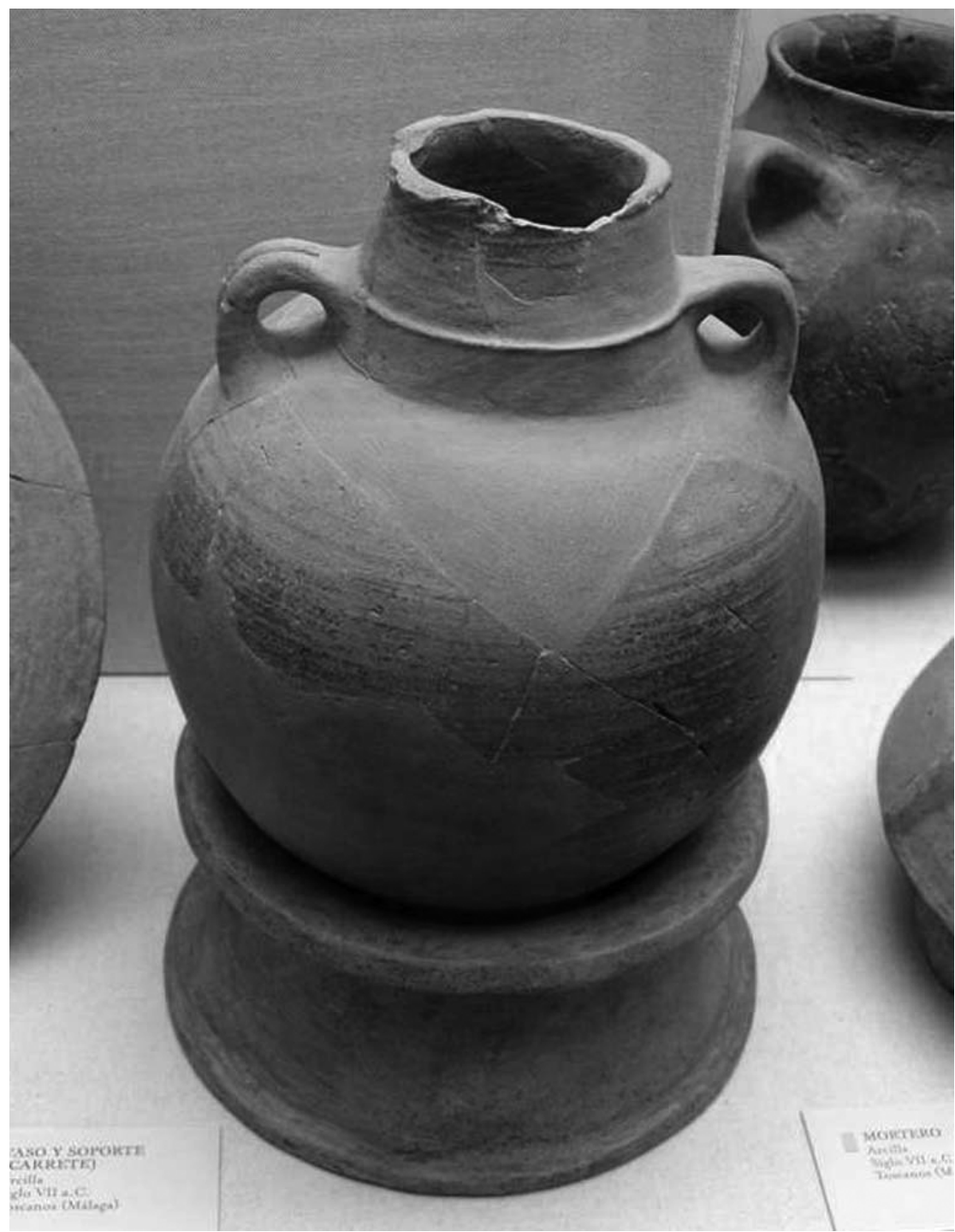

Fig. 1. Urna del tipo Cruz del Negro documentada en Toscanos. MAN (Foto: R. Rodríguez Muñoz).

totalmente diferente. En las necrópolis peninsulares de raigambre oriental, salvo contadas excepciones en momentos posteriores a su asimilación por parte de las poblaciones peninsulares, es extraño encontrar este tipo de urnas, por lo que el uso que de este objeto hicieron los fenicios difiere sensiblemente del original. En el yacimiento fenicio de Toscanos, por ejemplo, se ha documentado un ejemplar de este vaso casi completo que no había funcionado como urna funeraria (Schubart, Niemeyer y Pellicer 1969: 90-146; Schubart y Maas Lindeman 1984: 41- 
210). Del mismo modo, aunque con una cronología posterior al vaso del asentamiento malagueño, en el alfar de Camposoto (San Fernando, Cádiz), se han localizado algunos ejemplares que contenían restos de salazones en su interior, lo que sin duda nos apunta a un uso muy diversificado entre las poblaciones fenicias (Gago, Clavaín, Muñoz, Perdigones y Frutos 2000: 53).

Por tanto, nos encontramos con un objeto con una finalidad concreta que, tras su adopción como consecuencia de los contactos y la consiguiente mezcla de poblaciones, se emplea copiando e imitando su uso originario. Sabemos de su fabricación e imitación por los hallazgos de fragmentos de este tipo de recipientes, junto a pithoi y ánforas, en los hornos descubiertos en el Cerro de los Infantes (Pinos Puente), con una cronología de principios del siglo VII a.C., lo que indica una manufactura también local de este tipo de formas, cosa que por otro lado resulta obvia (Contreras, Carrión y Jabaloy 1983: 533-38). Así mismo, los ejemplares localizados en diversos puntos del Levante y Noreste peninsular, como las cuatro imitaciones realizadas a mano de la tumba 184 de Agullena 4 (Graells 2004: 64-65), el vaso del Coll del Moro de Gandesa (Rafel 1991: 103-109) o los localizados en les Moreres ${ }^{5}$ (González Prats 2002: 260-76), por citar tan sólo algunos ejemplos, plantean la posibilidad de que este tipo de recipiente, una vez instaurado como símbolo de distinción social, pudiera sufrir un proceso de estandarización por parte de algunas comunidades indígenas. Del mismo modo, los vasos aparecidos en necrópolis extremeñas, como la recientemente estudiada de Medellín (Almagro 2008: 631-54), apuntan a una producción estandarizada enfocada a un intenso comercio con esta zona.

\section{EL INTERCAMBIO DE OBJETOS COMO GERMEN DEL PROCESO DE MÍMESIS}

En general, para comprender el proceso de mímesis que se pudo haber producido en una realidad cultural concreta es de vital importancia el nuevo significado que adquiere un objeto cuando se traslada de un contexto -metrópoli o colonia- a otro nuevo - territorio 'colonizado'-. Ese objeto pasa a convertirse en un elemento de intercambio determinante en el proceso de relaciones sociales que se establece entre ambas culturas durante un periodo de tiempo concreto. Esto es lo que algunos autores han definido como 'teoría de la cultural material', en la que se estudia la naturaleza de los objetos y el modo en que los mismos crean o modifican las relaciones sociales (Gosden 2008: 138). Pienso que esta teoría puede muy bien corresponderse con el proceso cultural que se pudo haber producido en al-

${ }^{4}$ Esta tumba y las urnas que contenía fueron revisadas hace unos años por Graells, en cuyo trabajo se destaca la diferenciación de esta tumba con el resto de la necrópolis, afirmándose que el allí enterrado pudiera ser un personatge estranger introduït en les comunitats del nord-est de la península ibèrica $i$ del sud-est de França, fruit dels contactes amb els pobles colonitzadors de caràcter semita (2004: 74).

5 En el caso de esta necrópolis, recientemente se ha presentado un estudio en el que se propone que en ella se enterraría una clase dominante cuyo elevado estatus les facilitaría el empleo de este tipo de vasos en sus prácticas funerarias. En este sentido, véase Vives-Ferrándiz (2006: 196). 


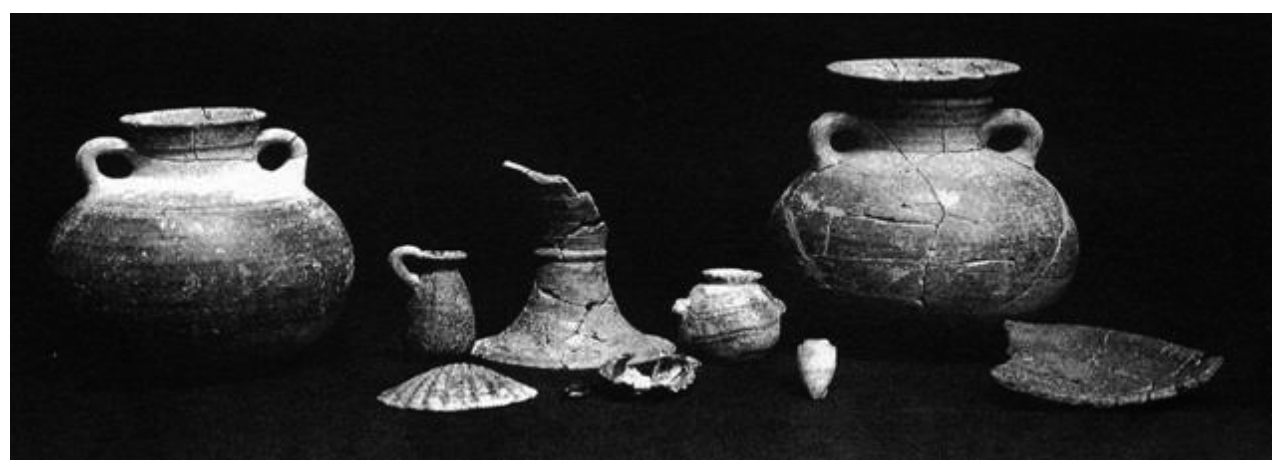

Fig. 2. Ajuar de la tumba 64 de la necrópolis de Las Cumbres (Ruiz Mata y Pérez 1995: lám. 3).

gunas zonas del ámbito peninsular hacia finales del siglo IX-VIII a.C. en torno a la adopción y posterior asimilación de la urna Cruz del Negro.

A tenor de lo anterior, tendríamos que interpretar que la adopción de este recipiente en concreto es susceptible de poder ser entendida desde la óptica del conocimiento por parte de las poblaciones autóctonas de que este vaso ya se empleaba en algunas necrópolis fenicias, con lo cual el proceso de mímesis debe interpretarse como una mera copia de ese recipiente, que se acepta, se asimila para un uso igual que el que se tenía en las necrópolis centromediterráneas, porque podíamos pensar que lo imitaron del contexto colonial próximo pero lo cierto es que las cronologías no casan. Los ejemplares documentados en las necrópolis fenicias se fechan en el siglo VI a.C., con lo que ya llevaban más de un siglo usándose por las poblaciones autóctonas.

Se podría abogar por la existencia de contactos entre comunidades peninsulares y centromediterráneas desde el siglo VIII a.C., aunque quizá también habría que pensar que, al igual que sucediera con la adopción de otros contenedores, como las ánforas o los pithoi, su asimilación y uso funerario se debió a que caló bien entre la población autóctona, hasta el punto de emplearlo en muchas ocasiones para contener las cenizas de sus muertos, ritual que ya realizaban anteriormente. Habría que plantearse entonces de dónde copiaron este comportamiento funerario, si entendemos que los diversos elementos orientales hallados en las tumbas locales se deben a la emulación de los objetos de ajuar de las nuevas poblaciones extranjeras -en este sentido, otro aspecto interesante de estudio podría ser si esta imitación llevó relacionada una nueva concepción de la muerte, es decir, si el comportamiento que copiaban lo hacían únicamente por las connotaciones sociales que pudiera tener en el seno de su comunidad o también por el entendimiento de la escatología oriental.

A pesar de esta cuestión, y retomando el objeto de nuestro estudio, no cabe duda de que la llegada de este vaso se debe relacionar con la presencia de los fenicios, ya que con anterioridad a los mismos no lo tenemos documentado en los cementerios hispanos. Posiblemente, su introducción deba asociarse a algún tipo 
de intercambio, en el que, al igual que algunas ánforas documentadas, contendrían algún tipo de producto foráneo. En cualquier caso, e independientemente de si conocían la realidad funeraria de otras zonas centromediterráneas o no, es menester aclarar que la imitación se produjo, a priori, en torno al uso y empleo de ese objeto en sí en las necrópolis indígenas, como signos de prestigio. Si no, no podemos explicar la presencia de uno de estos vasos realizado en bronce en la tumba n. ${ }^{\circ} 1$ de la necrópolis de La Joya (Garrido 1970: 65-66, lám. L; Orta y Garrido 1963: 10-36, figs. 4 y 5, láms. IV y V). No se realiza un recipiente en metal emulando un tipo en cerámica si no se considera éste como un bien de reafirmación del poder social ante la comunidad. En este sentido, de esta importante necrópolis proceden igualmente dos vasos del tipo aquí estudiado localizados entre los materiales de los enterramientos 12 y 16 respectivamente, lo que sin duda indica el carácter que tuvo este recipiente ${ }^{6}$ (Garrido y Orta 1978: 24-39, 48-63, figs. 11 y 28, láms. XV y XXXIII).

Esta consideración como símbolo de prestigio es básica para interpretar que, exceptuando algunas necrópolis como la de la Cruz del Negro o Las Cumbres, el porcentaje de este tipo de recipientes no es destacado dentro del conjunto funerario indígena, aunque sí es mucho más común y su número más elevado que en las necrópolis fenicias (Aubet 1978: 81-107; Ruiz Mata y Pérez 1995: 119-20). A pesar de ello, creo que un recipiente que se usa comúnmente en unas necrópolis bajo parámetros culturales similares llega a estandarizarse, por lo que deja de considerarse como bien de prestigio. A nivel de complejidad social, resultaría interesante poder relacionar su empleo con otros enterramientos coetáneos con el fin de determinar el posible estatus social de los que usaban estos recipientes para depositar sus cenizas, ya que como sabemos, también se han documentado otro tipo de contenedores en estas necrópolis en convivencia con las urnas Cruz del Negro. Actualmente los datos arqueológicos no nos permiten precisar este tipo de cuestiones.

Por otro lado, esta estandarización también llevaría aparejada la cuestión de averiguar sobre quién recaería la potestad de controlar y distribuir estas producciones, cuestión que, a la luz de los datos disponibles al respecto, es aún muy complicada, pero que se puede adivinar en la figura de algún jerarca de rango social elevado, aunque, como digo, es muy complicado debido al desconocimiento de la sociedad 'pre-fenicia' peninsular y su posterior transformación.

\section{INTERACCIÓN CULTURAL O HIBRIDACIÓN}

Con los datos de que disponemos, es sumamente complicado determinar si en los cementerios indígenas se produjeron uniones mixtas e hibridación. Posiblemente se llevaran a cabo, pero los datos arqueológicos no nos permiten concretar

${ }^{6}$ La boca y el borde de la urna de la tumba 16 difiere ligeramente del los tipos comunes. A pesar de ello, y teniendo en cuenta el grado de deterioro que presentaba el recipiente en el momento de su hallazgo, consideramos que la morfología general de la pieza obliga a su inclusión en este grupo. 
qué diferencias se pueden determinar como válidas para tratar de saber si un determinado enterramiento es autóctono o extranjero dentro de un conjunto general casi homogéneo. Por lo que respecta a la segunda cuestión, su escasez en las necrópolis de la cultura a la que se le asocia, en lo que al ámbito de la Península lbérica se refiere, nos anima a tratar el problema desde la perspectiva contraria, sin duda, a nuestro entender, más importante precisamente por la singularidad de los ejemplos constatados y porque sus resultados podrían indicar un modelo de relaciones e interacciones entre ambas culturas que difiere sensiblemente de la mera aculturación como consecuencia de la superioridad extranjera frente a la inferioridad autóctona.

Nueve parecen ser los ejemplares de este tipo de recipiente hallados en las necrópolis fenicias, a los que habría que sumar otro posible documentado en Los Monteros, Marbella, que se ha relacionado con contextos funerarios pero que, debido a la ausencia de más materiales que confirmen o no esta posibilidad, no adjudicamos como seguro (García 1998: 117-19). De ellos, tan sólo dos, localizados en Cádiz, han aparecido conteniendo cenizas, por lo que su función cineraria es clara. El resto se pueden considerar como vasos destinados a ofrendas, aunque los tres hallados en la necrópolis de Jardín, de los que únicamente se conserva el tercio superior de los mismos y cuyos dibujos invitan a pensar que se trata de este tipo de urnas, se evidenciaron en los niveles superiores y de relleno de las sepulturas, por lo que se cree que no formarían parte de su ajuar pero que posiblemente estuvieran relacionadas de algún otro modo con ellas (Rodríguez Muñoz 2006: 96101). En cualquier caso, lo que nos interesa recalcar es la escasez de estos vasos frente al elevado número presente en los enterramientos locales.

A tenor de esta cuestión, que se nos antoja fundamental, es menester indicar algunas consideraciones que son básicas para exponer nuestro planteamiento. Comenzando con la cronología, y como hemos apuntado unas líneas más arriba, no podemos precisar que en los cementerios indígenas se produjera una copia del modelo de enterramiento llevado a cabo por las poblaciones extranjeras ya que todos los ejemplares fenicios se fecharían en distintos momentos del siglo VI a.C., cuando ya llevan más de un siglo empleándose en distintas áreas funerarias peninsulares. Este dato es importantísimo porque nos sirve para reafirmar nuestra hipótesis planteada anteriormente: que las poblaciones locales existentes en la $\mathrm{Pe}$ nínsula Ibérica adoptaron este tipo de contenedor, dándole quizá un uso distinto al que inicialmente tuvo durante el proceso de intercambio por el que éste pasó a formar parte de la población local, asimilando y adaptando este objeto a la nueva realidad socio-cultural que surge necesariamente del encuentro entre culturas, y que en nuestro caso no sería una excepción.

Pero, además, esa adopción y posterior asimilación al comportamiento funerario ya existente, posiblemente continuador del anterior, supone el conocimiento, por parte de esas poblaciones, de esa nueva realidad material, que es recibida en un deseo de emular a las nuevas poblaciones llegadas de Oriente. El conocimiento de esa nueva realidad material se podría asociar quizá al entendimiento igualmente del comportamiento funerario de los extranjeros en otras zonas de la 
cuenca mediterránea, fruto, quizá, de contactos anteriores a la consolidación de los asentamientos fenicios peninsulares con otras áreas del Mediterráneo.

Por otro lado, a nivel cultural, esta escasa presencia de urnas Cruz del Negro puede ser entendida desde varios puntos de vista, si bien yo soy partidaria de relacionar su presencia con los contactos y las redes de intercambio creadas entre los fenicios y las poblaciones locales a partir de los siglos VIII-VII a.C., que en algunas zonas serían muy cercanas, hasta tal punto que algunos miembros de la población extranjera usarán este tipo de recipientes en sus enterramientos. Esta teoría ya fue apuntada por M. ${ }^{a} \mathrm{E}$. Aubet para el ejemplar hallado entre los materiales correspondientes a la necrópolis de Cortijo Montañez (Aubet 1986: 119).

Por otro lado, se podrían interpretar estos enterramientos como pertenecientes a indígenas enterrados en las necrópolis fenicias, hipótesis muy sugerente que creo debe ser matizada. Si bien en los últimos años se tiende a enfocar el fenómeno de la colonización desde el punto de vista de la coexistencia de culturas y la consiguiente hibridación entre las mismas, en el caso que yo presento aquí no estaría tan claro debido precisamente a la escasez de datos que permitan asegurar dicho proceso socio-cultural. Es muy factible que se produjeran uniones mixtas entre los extranjeros y los locales, pero el problema es que con la documentación disponible, por lo que respecta al sur peninsular, no se puede precisar el alcance de las mismas. Voy a poner un ejemplo a mi entender muy claro. Desde finales del siglo XIX se viene excavando en la ciudad de Cádiz, donde, hasta hace algo más de una década, sólo se habían documentado tumbas correspondientes a las fases fenicias y púnicas de la ciudad. Así, en la actualidad, y sin tener en cuenta los datos de las excavaciones efectuadas por D. Ramón Corzo entre 1979 y 1984, pendientes de ver la luz algún día, contamos con un registro funerario de considerable envergadura. Entre toda esta documentación tan sólo contamos con cuatro ejemplares conocidos de urnas Cruz del Negro, todos fechados en el siglo VI a.C. Resulta sumamente complejo interpretar estos datos, pero creo que no deben ser entendidos como consecuencia de un proceso de hibridación, cuando los que emplean este tipo de recipientes muestran valores y comportamientos similares al grupo con el que se entierra. Preferiría hablar mejor, en este sentido, de pautas funerarias particulares en el seno de la práctica generalizada fenicia, fruto de estos encuentros comerciales.

A ello habría que sumar la posible adscripción centromediterránea de una de ellas, que formaba parte del ajuar, junto a una lucerna de dos mechas, de un enterramiento en cista realizada en un solo bloque, con tapadera, que contenía en su interior una incineración, estando los materiales destinados a ofrendas depositados en el exterior y apoyados en la caja (Quintero 1926: 5-8; Rodríguez Muñoz 2006: 96-98). Este patrón de enterramiento se constata en la necrópolis fenicia de Mozia, donde los recipientes que se hallan adosados a las cistas, así como en el interior de las mismas, guardan claros paralelos con las urnas Cruz del Negro (Tusa 1978: 9-10).

Por tanto, quizá sería más sensato asociar los contados vasos funerarios con la influencia que también se pudo haber ejercido por parte de las poblaciones lo- 
cales del sur peninsular. Ello entronca necesariamente con el tema de la interacción cultural y la tan discutida aculturación. La presencia de este tipo de materiales en los cementerios fenicios, formando parte de su comportamiento funerario, indica que la influencia cultural no se dio únicamente desde la población extranjera hacia la población indígena, considerada siempre más atrasada que la anterior, sino que también se produjo en la dirección contraria, lo cual denota el grado de complejidad social que podrían tener las poblaciones autóctonas.

\section{CONCLUSIONES}

A tenor de lo anterior, creemos que bajo estos parámetros comentados se debería hablar, más que de colonización en sentido estricto, de colonialismo en un medio cultural compartido, usando una de las tres propuestas de colonialismo que define C. Gosden y que creo que encajaría, grosso modo, con la realidad socio-cultural surgida tras la presencia fenicia en la Península Ibérica (Gosden 2008: 57-100). Según este autor, dentro de un medio cultural compartido, se efectúan relaciones coloniales entre estado y formas de gobierno no estatales creadas en el seno de un medio natural compartido, quedando limitada la colonización al espacio por el que se extiende e intercambia la cultura. Bajo esta perspectiva, la interacción surgida en el SO. peninsular sería fruto del intercambio de la cultura indígena, heredera del Bronce Final, con la extranjera, a lo largo de un espacio en el que se coIonizaron los individuos a través de conceptos como la riqueza, los comportamientos comunes y la estética.

Así, se podrían interpretar los objetos orientales de diversa índole que, entendidos como objetos de lujo, son adoptados, emulando un comportamiento nuevo que se asume y adopta en gran medida, hasta convertirse en común, aunque sin olvidar la idiosincrasia propia de esa cultura. Igualmente, el uso de estos objetos, ya relacionados con la práctica indígena, por parte de algunos miembros foráneos debe entenderse como resultante de ese comportamiento común surgido en un espacio concreto, que quedaría definido por la propia extensión del mismo; es decir: allí donde se dejara notar su influencia, se podría hablar de comportamientos comunes.

\section{BIBLIOGRAFÍA}

ALMAGRO GORBEA, M. (1991), «La necrópolis de Medellín. Influencia fenicia en los rituales funerarios tartésicos", Trabajos del Museo Arqueológico de Ibiza 24, 233-252.

ALMAGRO GORBEA, M. dir. (2007), La Necrópolis de Medellín. I. La Excavación y sus Hallazgos, Madrid.

- (2008), La Necrópolis de Medellín. Il. Estudio de los Hallazgos, Madrid.

ARANEGUI GASCÓ, C. (1980), "Contribución al estudio de las urnas de tipo Cruz del Negro", Saguntum-PLAV 15, 99-118.

ARRIBAS, A. y WILKINS, J. (1969), «La necrópolis fenicia del Cortijo de las Sombras (Frigiliana, Málaga)», Pyrenae 5, 185-256.

AUBET, M. E. (1976), «La cerámica púnica de Setefilla», Studia Archaeologica 42, 5-34. 
- (1978), «La cerámica a torno de la Cruz del Negro (Carmona, Sevilla)», Ampurias 38-40, 267-287.

- (1986), «Contactos culturales entre el Bajo Guadalquivir y el Noroeste de África durante los siglos VII y VI a.C.», Gli Intercambi Culturali e Socio-economici fra I'Africa Settentrionale e I'Europa Mediterranea. Atti del Congresso Internazionale di Amalfi, 5-8 diciembre 1983, Nápoles, 109-144.

BELÉN, M. y PEREIRA SIESO, J. (1985), “Cerámicas a torno con decoración pintada en Andalucía», HA 7, 307-359.

BELÉN, M.; GIL, M. S.; LINEROS, R. y PUYA, M. (1987), «Excavaciones en el Campo de las Canteras (Carmona, Sevilla). El túmulo A», XVIII CNA, 535-553.

CINTAS, P. (1950), Céramique Punique, Túnez.

CONTRERAS CORTÉS, F.; CARRIÓN MÉNDEZ, F. y JABALOY SÁNCHEZ, E. (1983), «Un horno de alfarero protohistórico en el Cerro de los Infantes (Pinos Puente, Granada)", Crónica del XVI Congreso Arqueológico Nacional, Cartagena, 533-538.

FERNÁNDEZ FLORES, A. y RODRÍGUEZ AZOGUE, A. (2007), «Vida y muerte en la llipa tartésica», en Ferrer Albeada, E.; Fernández Flores, A.; Escacena Carrasco, J. L. y Rodríguez Azogue, A. eds., llipa Antiqva. De la Prehistoria a la Época Romana, Sevilla, 69-92.

GAGO VIDAL, M. H.; CLAVAÍN GONZÁLEZ, I.; MUÑOZ VICENTE, A.; PERDIGONES MORENO, L. y FRUTOS REYES, G. (2000), «El complejo industrial de salazones de Camposoto, San Fernando (Cádiz): estudio preliminar», Habis 31, 37-61.

GARCÍA ALFONSO, E. (1998), «Dispersión de los vasos tipo Cruz del Negro en la Alta Andalucía: el ejemplar de Marbella (Málaga)», Homenaje al Profesor Carlos Posac Mon 1, 115-129.

GARRIDO ROIZ, J. P. (1970), Excavaciones en la Necrópolis de La Joya, EAE 71, Madrid.

GARRIDO ROIZ, J. P. y ORTA GARCÍA, E. (1978), Excavaciones en la Necrópolis de La Joya, Huelva, EAE 96, Madrid.

GIL DE LOS REYES, S. y PUYA GARCÍA DE LEÁNIZ, M. (1995), «Excavaciones en la necrópolis de la Cruz del Negro (Carmona, Sevilla)», Actes du IIle Congrès Internacional des Études Phéniciennes et Puniques II, Túnez, 83-87.

GONZÁLEZ RODRÍGUEZ, R.; BARRIONUEVO CONTRERAS, F. y AGUILAR MOYA, L. (1993), "Mesa de Asta, un centro indígena tartésico en los esteros del Guadalquivir», Tartessos 25 años después (1968-1993), Jerez, 215-237.

GONZÁLEZ PRATS, A. (2002), La Necrópolis de Cremación de Les Moreres (Crevillente, Alicante, España) (siglos IX-VII a.C.), Alicante.

GOSDEN, C. (2008), Arqueología y Colonialismo. El Contacto Cultural desde 5000 a.C. hasta el Presente, Barcelona.

GRAELLS I FABREGAT, R. (2004), «Indicis d'emergència aristocràtica al registre funerari del nord-est peninsular. La tomba Agullana 184», RAP 14, 61-83.

JIMÉNEZ ÁVILA, F. J. (2002), La Toréutica Orientalizante en la Península Ibérica, Madrid.

ORTA, E. M. y GARRIDO, J. P. (1963), La Tumba Orientalizante de La Joya, Huelva, Huelva.

QUINTERO ATAURI, P. (1926), Excavaciones en Extramuros de Cádiz, MJSEA 84, Madrid.

RAFEL, N. (1991), La Necrópolis del Coll del Moro de Gandesa. Els Materials, Tarragona.

RODRÍGUEZ MUÑOZ, R. (2006), «Estudio sobre la presencia y el uso de las urnas Cruz del Negro en las necrópolis fenicias de Andalucía», Saguntum-PLAV 38, 93-108.

RUIZ MATA, D. (1987), «Excavación del túmulo 1 de la necrópolis de Las Cumbres (Puerto de Santa María, Cádiz), 1985», Anuario Arqueológico de Andalucía/1985, Sevilla, 158160.

RUIZ MATA, D. y PÉREZ, C. (1989), «El túmulo 1 de la necrópolis de 'Las Cumbres' (Puerto de Santa María, Cádiz)», Arqueología protohistórica del Bajo Guadalquivir, Sabadell, 287-295.

- (1995), El Poblado Fenicio del Castillo de Doña Blanca (El Puerto de Santa María, Cádiz), Cádiz. 
SÁNCHEZ ANDREU, M. y LADRÓN DE GUEVARA, I. (2000), «Necrópolis del Camino: sepulturas tipo 'Cruz del Negro' en Bencarrón (Sevilla)», Actas del IV Congreso internacional de Estudios Fenicios y Púnicos IV, Cádiz, 1895-1902.

SCHUBART, H.; NIEMEYER, H. G. y PELLICER CATALÁN, M. (1969), Toscanos. La Factoría Paleopúnica en la Desembocadura del rio Vélez, EAE 66, Madrid.

SCHUBART, H. y MAAS-LINDEMANN, G. (1984), «Toscanos. El asentamiento fenicio occidental en la desembocadura del río Vélez. Excavaciones de 1971», Noticiario Arqueológico Hispánico 18, 41-210.

TORRES ORTIZ, M. (1999), Sociedad y Mundo Funerario en Tartessos, Madrid.

TUSA, V. (1978), «Relazione preliminare degli scavi eseguiti a Mozia negli anni 1972, 1973, 1974», Mozia 9, 7-98.

VIVES-FERRÁNDIZ SÁNCHEZ, J. (2006), Negociando Encuentros. Situaciones Coloniales e Intercambios en la Costa Oriental de la Península Ibérica (ss. VIII-VI a.C.), Cuadernos de Arqueología Mediterránea 12, Madrid. 